\title{
Hydrogen storage of nanocrystalline Mg-Ni alloy processed by equal-channel angular pressing and cold rolling
}

\author{
Ádám Révész ${ }^{\mathrm{a}, 1}$, Marcell Gajdics ${ }^{\mathrm{a}}$, Lajos K. Varga ${ }^{\mathrm{b}}$, György Krállics ${ }^{\mathrm{c}}$, László Péter ${ }^{\mathrm{b}}$, \\ Tony Spassov ${ }^{\mathrm{d}}$
}

${ }^{\mathrm{a} D e p a r t m e n t ~ o f ~ M a t e r i a l s ~ P h y s i c s, ~ E o ̈ t v o ̈ s ~ U n i v e r s i t y, ~ B u d a p e s t . ~ H-1518, ~ P . O . B . ~ 32, ~}$ Budapest, Hungary

${ }^{\mathrm{b}}$ Institute for Solid state Physics and Optics, Wigner Research Center for Physics, Hungarian Academy of Sciences. H-1525, P.O.B. 49, Budapest, Hungary

${ }^{c}$ Department of Materials Science and Engineering, Budapest Univeristy of Technology and Economics. H-1111 ,Bertalan L. str. 7. Budapest, Hungary

${ }^{\mathrm{d}}$ Department of Chemistry, University of Sofia "St.K1.Ohridski". 1 J.Bourchier str., 1164 Sofia, Bulgaria

\begin{abstract}
Ball-milled nanocrystalline $\mathrm{Mg}_{2} \mathrm{Ni}$ powders were subjected to intense plastic straining by cold rolling or equal-channel angular pressing. Morphological and microstructural evolution during these processes has been investigated by scanning electron-microscopy and X-ray diffraction line profile analysis, respectively. Complementary hydrogen absorption experiments in a Sieverts' type apparatus revealed that there exists some correlation between the micro- and nanostructure and hydrogen storage properties of the severely deformed materials.
\end{abstract}

Keywords: Hydrogen storage; Mg-based; Microstructure; Severe plastic deformation; Cold rolling, Equal-channel angular pressing

\footnotetext{
${ }^{1}$ Corresponding author. E-mail: reveszadam@ludens.elte.hu Tel.: +36-1-372-2823, Fax: +36-1-372-2811
} 


\section{Introduction}

Hydrogen storage has been attracting considerable interest for couple of decades, since hydrogen as an energy carrier can provide several times more power per mass compared to conventional fossil resources $\left(120 \mathrm{MJkg}^{-1}\right)$ [1]. Hydrogen is difficult to be stored in the gas state, however, efficient storage can be achieved in the solid-state by simple and complex metallic hydrides, since they show remarkable gravimetric and volumetric storage capacities [2]. Among them, one of the most promising candidate is magnesium, since it exhibits very high storage capacity (7.6 wt.\%), lightweight and moderate cost [3]. Unfortunately, the high stability of the hydride phase and sluggish $\mathrm{Mg} \leftrightarrow \mathrm{MgH}_{2}$ kinetics are critical obstacles against the use of commercial $\mathrm{MgH}_{2}$ in wide-spread practical applications. High energy ball milling (HEBM) processing technique has been applied to produce $\mathrm{Mg}$ powders or $\mathrm{Mg}$-based composites since this method easily lead to simultaneous particle size and crystallite size reduction [4-8], which enhances the hydrogen sorption kinetics significantly due to the increased volumetric density of grain boundaries and lattice defects [9-10]. Milling metal or metal-oxides catalyst powders with magnesium results in a fine and homogeneous dispersion of the additives, accompanied with the thermodynamical destabilization of $\mathrm{MgH}_{2}[3,8,11]$. It was found that the addition of nickel to magnesium results in improved thermodynamics and hydrogen sorption kinetics [12-13]. According to the Mg-Ni binary phase diagram, the two elements are mutually insoluble in the other, while there exists two intermetallic compounds: $\mathrm{Mg}_{2} \mathrm{Ni}$ and $\mathrm{MgNi}_{2}$, however, only the former exhibits significant hydrogen storage capacity (3.62 wt.\%) [14]. Under specific milling conditions and proper composition of $\mathrm{Mg}$-Ni powder blends, the $\mathrm{Mg}+\mathrm{Ni} \rightarrow \mathrm{Mg}_{2} \mathrm{Ni}$ solid-state chemical reaction can take place [15-16].

Despite the plenty of benefits of HEBM, there are several disadvantages in powder processing by mechanical milling, including surface oxidation, energy consumption, potential fire risk and expensiveness of industrial level production. These disadvantages of HEBM to prepare Mg-based alloys for H-storage can extensively be eliminated by severe plastic deformation (SPD) techniques, including high-pressure torsion (HPT), cold rolling (CR) and equal-channel angular pressing (ECAP). These methods originally were invented for producing porosity and impurity-free bulk nanocrystalline samples [17-18]. The SPD techniques have numerous benefits, i.e. the process is less intensive than HEBM, scaling up to larger quantities at a lower cost has larger potential, impurity concentration is lower and it causes less safety concerns [19]. 
Kusadome at al. have demonstrated that intensive straining by HPT can promote hydrogen uptake in an otherwise non-absorbing compound such as $\mathrm{MgNi}_{2}$ [20]. When pure $\mathrm{Mg}$ is subjected to HPT, the hydrogen absorption becomes prominent and the sorption rate is significantly increased [21]. Révész et al. pointed out that the abundant lattice defects, i.e. dislocations, generated during HPT play a crucial role in the increased amount of absorbed hydrogen [16].

The beneficial H-sorption kinetics of cold rolled Mg-based alloys was systematically reviewed by Huot [22]. By comparing the kinetics of $\mathrm{MgH}_{2}$ doped with transition metal oxides as catalysts, it was found that cold rolled samples possess relatively slower dehydrogenation kinetics compared to the milled counterparts, since atomic mixing is more difficult by CR than by HEBM [23]. Nevertheless, the potential advantage of CR is that all operation can be made in air, at least for small number of rolls the hydrogen capacity loss was found to be minimal [24]. The combination of melt spinning and cold rolling on a $\mathrm{Mg}-\mathrm{Ni}$ alloy with refined microstructure and texture led to a large defect density and attractive $\mathrm{H}$ sorption kinetics after activation [25].

Equal-channel angular pressing was successfully applied to produce bulk Mg-based hydrides with enhanced hydrogen storage properties by Skripnyuk et al [26]. Multi-pass ECAP process of an $\mathrm{Mg}-\mathrm{Ni}$ alloy at elevated temperature results in a two phase $\left(\mathrm{Mg}+\mathrm{Mg}_{2} \mathrm{Ni}\right)$ microstructure [27]. The increase of the equilibrium hydrogen pressure is associated with the chemical inhomogeneity of the sample. The combination of ECAP and HEBM on the commercial Mgbased ZK60 alloy eliminates the pressure hysteresis and also increases the desorption pressure [19]. Furthermore ECAP can produce special features for hydrogen sorption such as preferential textures [28].

In the present study, we demonstrate the evolution of the microstructure of ball-milled $\mathrm{Mg}_{2} \mathrm{Ni}$ followed by CR or ECAP by applying the Convolutional Whole Profile Fitting (CMWP) algorithm of X-ray line profiles. A correlation between the microstructure and hydrogen sorption will be highlighted. 


\section{Experimental}

\subsection{Powder processing}

Ball milling blend of commercial polycristalline $\mathrm{Mg}$ and Ni powders (supplied by SigmaAldrich; purity 99.9\%) was carried out using a high-energy SPEX 8000 Mixer Mill with a nominal composition $\mathrm{Mg}_{75} \mathrm{Ni}_{25}$. The vial was filled with 10 1/4" stainless steel balls. The balls to sample weight ratio was 10:1. The filling of the milling container always performed in an argon-flushed glove box. Milling time was $10 \mathrm{~h}$, which ensures the formation of nanocrystalline $\mathrm{Mg}_{2} \mathrm{Ni}$ powder through the $2 \mathrm{Mg}+\mathrm{Ni} \rightarrow \mathrm{Mg}_{2} \mathrm{Ni}$ solid state reaction [16]. For further SPD processing by ECAP and CR, the milled $\mathrm{Mg}_{2} \mathrm{Ni}$ powders were compacted into pellets with a diameter of $10 \mathrm{~mm}$ and height of $4 \mathrm{~mm}$ under $1 \mathrm{GPa}$ of hydraulic pressure.

\subsection{Equal-channel angular pressing}

For ECAP processing, 10-12 as-compacted $\mathrm{Mg}_{2} \mathrm{Ni}$ billets were stuck and encapsulated together in a cylindrical aluminum tube (diameter $16 \mathrm{~mm}$, height $1000 \mathrm{~mm}$ ). The ECAP procedure have been performed at room temperature using a solid die with an internal channel angle of $\Phi=90^{\circ}$ and an angle of the outer curvature of the two parts of the die $\psi=30^{\circ}$ [18]. For our experiments, repetitive pressings (ECAP 2x and ECAP 6x) have been performed at ambient temperature using route " $\mathrm{A}$ " without any rotation between the subsequent passages [18]. For further characterization slices of the tube were mechanically polished.

\subsection{Cold rolling}

Prior to cold rolling experiments, the as-pressed $\mathrm{Mg}_{2} \mathrm{Ni}$ pellets were sealed in a copper tubular with equal height in order to ensure a fixed geometry during the process. Subsequently, CR was performed at room temperature by inserting the billets between two stainless steel plates using a conventional rolling mill with a diameter of $100 \mathrm{~mm}$. to prevent contamination from the rolls. Experiments were conducted in air for CR 1x CR 4x and CR 10x repetitive passes.

\subsection{Microstructural characterization}

Microstructural studies were performed by X-ray powder diffraction (XRD) with $\mathrm{Cu}-\mathrm{K} \alpha$ radiation on a Philips X'pert powder diffractometer in $\theta-2 \theta$ geometry. The X-ray diffraction patterns have been evaluated by the CMWP fitting procedure developed by Ungár and coworkers, which includes the direct fit of the whole measured X-ray diffractogram by the sum 
of background, theoretically constructed profile functions and measured instrumental profile [30]. The line profile analysis provides microstructural parameters, i.e. average coherent crystallite size (D), median $(\mathrm{m})$ and variance $(\sigma)$ of crystallite size distribution, density of lattice defects. More details of the fitting algorithm [29] and its application to ball-milled Mgbased powders can be found elsewhere [30-31], while the program can be downloaded from its web interface [32].

Morphology was studied on a FEI QUANTA 3D dual beam scanning electron microscope (SEM). Local composition was quantitatively determined by energy dispersive X-ray (EDX) analysis.

\subsection{Hydrogen sorption kinetics}

Hydrogenation kinetic curves of the CR and ECAP processed samples were measured by a Sieverts'-type apparatus (PCT) at $573 \mathrm{~K}$. The initial hydrogen pressure was set as $1 \mathrm{MPa}$. For details, see [16].

\section{Results and discussion}

Figure 1 presents the microstructural evolution of the $\mathrm{Mg}_{2} \mathrm{Ni}$ billets during ECAP and CR processing. The diffractograms of the milled and as-pressed samples is also shown for comparison. In general, each pattern is dominated by the peaks of hexagonal $\mathrm{Mg}_{2} \mathrm{Ni}$ (JCPDS $35-1225 ; \mathrm{a}=5.21 \AA$; $\mathrm{c}=13.323 \AA$ ) compound phase, however, small quantity of fcc $\mathrm{Ni}$ (JCPDS 45-1027) is also present. The presence of unreacted $\mathrm{Ni}$ is not unusual in HEBM Mg$\mathrm{Ni}$ powders, albeit the starting powder blend contains excess $\mathrm{Mg}$ compared to the stochiometric concentration. [16,31]. One can also notice that the as-milled powder contains some portion of $\mathrm{MgO}$, however its intensity remains practically constant after the hydrostatic compression. As the ECAP procedure introduces severe plastic deformation, the Bragg peaks of the $\mathrm{Mg}_{2} \mathrm{Ni}$ undergoes an insignificant line broadening while a close inspection reveals their relative intensities remains the same, corresponding to a moderate grain refinement and the lack of texture, respectively. At the same time, the $\mathrm{MgO}$ content seems to be constant. Some peaks of fcc $\mathrm{Al}$ can be recognized on the patterns of the ECAP samples, which originates from $\mathrm{Al}$ particles that have been pressed into the $\mathrm{Mg}_{2} \mathrm{Ni}$ billets during the process. Very similar behavior is realized from the plots corresponding to the cold rolled samples, i.e. slight 
line broadening and the lack of any growth of the $\mathrm{MgO}$ phase. Reflections from the copper ring is also noticed for the CR 10x specimen

The fitted diffractogram by the CMWP method corresponding to the ECAP 6x sample is presented in Fig. 2. The difference between the measurement and fit is also plotted. Table 1 summarizes the key microstructural parameters obtained from the CMWP analysis for the dominant $\mathrm{Mg}_{2} \mathrm{Ni}$ phase of ECAP and CR samples. As listed, the value of $\mathrm{m}$ remains in the order of the initial value $(4.7 \mathrm{~nm})$ after pressing and ECAP processing. At the same time, $\sigma$ obeys a slight monotonous increase from 0.68 to 0.74 after two ECAP passes, while final value $(\sigma=0.64)$ indicates that the crystallite size distribution of the ECAP 6x sample is similar to that of the HEBM state. Figure 3 a presents the corresponding normalized distribution functions determined from the CMWP analysis. It is evident from the plots that the hydraulic pressure on the as-milled $\mathrm{Mg}_{2} \mathrm{Ni}$ powders as well as the subsequent ECAP processing have only a marginal effect on the microstructure. The variation of the volume averaged coherent domain size $\left(\mathrm{D}=\mathrm{m} \exp \left(2.5 \sigma^{2}\right)\right)$ during the ECAP process can also be monitored in Table 1 and plotted in the inset of Fig. 3a. The value obtained for the $\mathrm{Mg}_{2} \mathrm{Ni}$ compound phase characterizing the as-milled powder $(D=15 \mathrm{~nm})$ and the as-pressed billet $(D=18) \mathrm{nm}$ is in line with literature data [16,33]. With increasing ECAP deformation, only a moderate crystallite size reduction is noticed $(\mathrm{D}=13 \mathrm{~nm}$ for ECAP $6 \mathrm{x})$.

The CMWP analysis of the X-ray scattering experiments of the cold rolled specimens indicates a slightly different evolution. Although, the particle size histograms of the CR $1 \mathrm{x}$ sample exhibits very similar median $(\mathrm{m}=4.9 \mathrm{~nm})$ and variance $(\sigma=0.71)$ resulting in similar final crystallite size $(\mathrm{D}=17 \mathrm{~nm})$ as it was already achieved after pressing the as-milled powder into pellets (Fig. $3 \mathrm{~b}$ and Table 1), a considerable broadening of the crystallite-size distribution is realized after $4 \mathrm{CR}$ passes possessing an increased variance of $\sigma=0.83$, which corresponds to a relatively more heterogeneous microstructure. At the same time a considerable recrystallization takes place, since the volume averaged crystallite size increases rapidly up to $\mathrm{D}=37 \mathrm{~nm}$. Interestingly, prolonged $\mathrm{CR}$ processing retains the microstructural homogeneity after 10 passes $(\sigma=0.73)$ and the finer average coherent crystallite size $(D=18 \mathrm{~nm})$, see the inset in Fig. 3b. This phenomenon most probably can be attributed to the varying mechanical properties (harness, strength) of the deformed samples.

The backscattered SEM image of the as-pressed $\mathrm{Mg}_{2} \mathrm{Ni}$ pellet is characterized by compact individual powder particles with sharp edges of an average particle size of $\sim 3 \mu \mathrm{m}$ (Fig. $4 \mathrm{a}$ ). 
Quantitative EDX analysis revealed that the average concentration of the pellet is slightly enriched in $\mathrm{Ni}\left(\mathrm{Mg}_{73} \mathrm{Ni}_{27}\right)$ compared to the nominal composition. The presence of the almost pure Ni bright particle in the center of the micrograph is in line with the XRD experiments (see Fig. 1). Figure $4 \mathrm{~b}$ and $4 \mathrm{c}$ corresponding to the CR $1 \mathrm{x}$ and CR $4 \mathrm{x}$ samples, respectively, exhibit slightly larger powder agglomerates $(\sim 5-20 \mu \mathrm{m}$ in size), however, a substantial densification takes place, while robust cracks and paths dominate the images, which presumably can accelerate the hydrogen sorption. Notwithstanding the rather similar nanostructure obtained by the CMWP analysis between the CR and ECAP processed samples, SEM images indicate considerable differences on the micron scale. The original morphology of the milled powders underwent drastic changes during the ECAP processing, i.e. a significant compaction occurs and the individual powder particles cannot be distinguished corresponding to the constrained geometry of the processing method (Figs. 4d and 4e) [18].

Figure 5 presents the hydrogenation kinetic curves obtained at $573 \mathrm{~K}$ for the ECAP and CR specimens. Both series of samples absorb hydrogen without activation, while the maximum capacity is achieved already after one hydrogenation-dehydrogenation cycles. The maximum amount of absorbed hydrogen is about $1.5 \mathrm{wt}$ \% for both the ECAP 2x and ECAP 6x samples. This value is considerably lower than that of the as-milled $\mathrm{Mg}_{2} \mathrm{Ni}$ powder [16]. As one can notice, the initial absorption kinetics is fast for both samples, reaching the 50 percent of the maximum hydrogen amount within less than $300 \mathrm{~s}$. The initial absorption rate, determined as the derivative of the hydrogen uptake curve at the start of the reaction, also presents similar behavior of the two ECAP samples. Remarkably higher hydrogen capacity is observed when the as-milled $\mathrm{Mg}_{2} \mathrm{Ni}$ powder is subjected to the $\mathrm{CR}$ deformation. Although the hydrogen uptake during the first sorption cycle (1.6 wt.\%) is similar to the value achieved by ECAP, it reaches 2.4 wt.\% after $\mathrm{CR} 4 \mathrm{x}$ which then levels off after prolonged processing. At the same time, the kinetics of the $\mathrm{CR} 4 \mathrm{x}$ sample exhibits outstanding performance, reaching $7.6 \times 10^{-3}$ wt. $\% \mathrm{~s}^{-1}$ at the beginning of the absorption. Figures $6 \mathrm{a}$ and $6 \mathrm{~b}$ summarizes the H-storage behavior of the $\mathrm{Mg}_{2} \mathrm{Ni}$ alloys obtained by the combination of different SPD routes. For comparison, data corresponding to HPT processing are also hired from Ref. [16]. As one can realize from Fig 6a, HPT noticeably improves the hydrogen storage capacity of ball-milled $\mathrm{Mg}_{2} \mathrm{Ni}$ powder, exhibiting the most outstanding storage performance among all the investigated SPD samples. The obtained capacity (3 wt.\%), corresponding to the composition of $\mathrm{Mg}_{2} \mathrm{NiH}_{3.3}$, indicates nearly complete hydriding [16]. This increase in the hydrogen absorption was attributed to the smaller crystallite, intense straining and/or lattice defects 
generated during the heavy shear deformation during HPT [16]. Nevertheless, ECAP significantly deteriorates the storage performance of $\mathrm{HEBM} \mathrm{Mg}_{2} \mathrm{Ni}$ powders, the maximum hydrogen capacity decreases by about 40 percent after 2 or 6 passes through the die. Similar reduction is noticed after one pass of cold rolling, however, the initial value $(2.4 \mathrm{wt} . \%)$ is retained after $10 \mathrm{CR}$ passes. Although very similar nanostructure have been created by HPT [16], ECAP 6x and CR 10x of HEBM $\mathrm{Mg}_{2} \mathrm{Ni}$ alloys, the storage performance of the HPT samples is far the best. Nevertheless, if we compare the equivalent strains achieved by CR $(\gamma=2.4$ after 10 passes [34]), ECAP $(\gamma=10.2$ after 6 passes [18]) and HPT $(\gamma \sim 300$ after 5 rotations [16]), a massive difference is realized. Besides the enormous straining achieved by HPT, the deformation mode itself, including severe shear can also be accountant for the increased capacity through the extensive formation of one dimensional lattice defects [16], which can also act as possible hydrogen absorption sites.

If we have a closer look on the data points describing how fast the kinetics are, different conclusions can be justified. In general, the initial absorption rate of the as-milled powder $\left(5.3 \times 10^{-3} \mathrm{wt} \% \mathrm{~s}^{-1}\right)$ is reduced after all kind of SPD processes, i.e. $3.0 \times 10^{-3} \mathrm{wt} . \% \mathrm{~s}^{-1}$ after HPT, $3.7 \times 10^{-3} \mathrm{wt}^{2} \% \mathrm{~s}^{-1}$ after 2 ECAP passes and $2.3 \times 10^{-3} \mathrm{wt}^{\circ} \% \mathrm{~s}^{-1}$ after $1 \mathrm{CR}$ pass (see Fig. $6 \mathrm{~b}$ ). This phenomenon can be related to the "thickness effect" [28], that takes place the extensive compaction during these bulk processing techniques, hampering the fast diffusion channels for the hydrogen atoms along the denser grain boundaries. The marked increase in the kinetics of the CR 4x sample (see Figs. 5 and $6 \mathrm{~b}$ ) is an interesting phenomenon. According to the SEM experiments, the morphological differences between the CR 1x and CR 4x samples are marginal (see Fig. 4), yet the CMWP analysis indicated a microstructural heterogeneity on the nanoscale. Seemingly, the diverse subgrain structure within the individual powder particles can significantly increase the hydrogen uptake. When the $\mathrm{Mg}_{2} \mathrm{Ni}$ particles are again built up from nanosized coherent crystallite domains of equal size after $10 \mathrm{CR}$ passes (see Fig. 3b), the $\mathrm{H}$-absorption rate levels off at the value of the HEBM sample.

\section{Conclusions}

Ball-milled nanocrystalline $\mathrm{Mg}_{2} \mathrm{Ni}$ powders were subjected to intense plastic straining by cold rolling and equal-channel angular pressing. The CMWP analysis of X-ray line profiles has shown that ECAP deformation on the HEBM powders results in a moderate crystallite size reduction $(\mathrm{D}=13)$, while the distribution of the coherently scattering domains preserves its 
homogeneity. On contrary, the CR specimens undergoes different evolution, i.e. considerable broadening of the crystallite-size distribution is realized after $4 \mathrm{CR}$ passes accompanied with a rapid crystallite size increase up to $\mathrm{D}=37 \mathrm{~nm}$. SEM experiments of the ECAP samples confirmed considerable compaction, while the original powder particles still can be resolved after CR. Hydrogen absorption measurements revealed that both ECAP and CR reduces the storage capacity of the $\mathrm{HEBM} \mathrm{Mg}_{2} \mathrm{Ni}$ powders, however, the broader crystallite size distribution of the CR 4x sample is coupled with an enhanced kinetics. In summary, it is concluded that the amount of equivalent strain of different SPD techniques, the deformation geometry, and the resulting nanostructure and microstructure are together responsible for the $\mathrm{H}$-storage behavior of nanocrystalline $\mathrm{Mg}_{2} \mathrm{Ni}$ alloys.

\section{Acknowledgements}

Á. R. is indebted for the János Bolyai Research Scholarship of the Hungarian Academy of Sciences. T.S. is grateful to the FP7 project Beyond Everest. The authors appreciate the support of G. Varga in the SEM experiments. We acknowledge the support of the COST Action MP1103. 


\section{References}

[1] Rönnebro ECE, Majzoub EH. Recent advances in metal hydrides for clean energy application. MRS Bulletin 2013;38:452-8.

[2] Schlapbach L, Züttel A. Hydrogen-storage materials for mobile applications. Nature 2011;414:353-8.

[3] Varin RA, Czujko T, Wronski ZS. Nanomaterials for Solid State Hydrogen Storage. New York: Springer Science; 2009.

[4] Zaluski L, Zaluska A, Tessier P, Ström-Olsen JO, Schulz R. Nanocrystalline Hydrogen Absorbing Alloys. Mater Sci. Forum 1996;225:853-8.

[5] Liang G, Boily S, Huot J, Van Neste A, Schulz R. Mechanical alloying and hydrogen absorption properties of the Mg-Ni system. J. Alloys Compds 1998;276:302-6.

[6] Fuster V, Urretavizcaya G, Castro FJ. Characterization of $\mathrm{MgH}_{2}$ formation by low-energy ball-milling of $\mathrm{Mg}$ and $\mathrm{Mg}+\mathrm{C}$ (graphite) mixtures under $\mathrm{H}_{2}$ atmosphere. $\mathrm{J}$ Alloys Compds 2009;481:673-80.

[7] Fátay D, Révész Á, Spassov T. Particle size and catalytic effect on the dehydriding of $\mathrm{MgH}_{2}$. J Alloys Compds 2005;399:237-41.

[8] Oelerich W, Klassen T, Bormann R. Metal oxides as catalysts for improved hydrogen sorption in nanocrystalline Mg-based materials. J Alloys Compds 2001;315:237-242.

[9] Révész Á, Fátay D, Spassov T. Microstructure and hydrogen sorption kinetics of $\mathrm{Mg}$ nanopowders with catalyst. J Alloys Compd 2007;434-435:725-8.

[10] Fátay D, Spassov T, Delchev P, Ribárik G, Révész Á. Microstructural development in nanocrystalline $\mathrm{MgH}_{2}$ during $\mathrm{H}$-absorption/desorption cycling. Int $\mathrm{J}$ Hydrogen Energy 2007;32:2914-9.

[11] Vincent SD, Lang J, Huot J. Addition of catalysts to magnesium hydride by means of cold rolling. J Alloys Compds 2012;512:290-5.

[12] Zeng K, Klassen T, Oelerich W, Bormann R. Thermodynamic analysis of the hydriding process of Mg-Ni alloys. J Alloys Compds 1999;283:213-24. 
[13] Simchi H, Kaflou A, Simchi A. Synergetic effect of $\mathrm{Ni}$ and $\mathrm{Nb}_{2} \mathrm{O}_{5}$ on dehydrogenation properties of nanostructured $\mathrm{MgH}_{2}$ synthesized by high-energy mechanical alloying. Int $\mathrm{J}$. Hydrogen Energy 2009;34:7724-30.

[14] Massalski TB. Ed. Binary Alloy Phase Diagram. American Society for Metals 1986.

[15] Shao H, Asano K, Enoki H, Akiba E. Preparation and hydrogen storage properties of nanostructured Mg-Ni BCC alloys. J Alloys Compd 2009;477:301-6.

[16] Révész Á, Kánya Zs, Verebélyi T, Szabó PJ, Zhilyaev AP, Spassov T. The effect of high-pressure torsion on the microstructure and hydrogen absorption kinetics of ball-milled $\mathrm{Mg}_{70} \mathrm{Ni}_{30}$. J Alloys Compds 2010;504:83-8.

[17] Valiev RZ, Ishlamgaliev RK, Alexandrov IV. Bulk nanostructured materials from severe plastic deformation. Prog Mater Sci 2000;45:103-89.

[18] Valiev RZ, Langdon TG. Principles of equal-chanel angular pressing as a processing tool for grain refinement. Prog Mater Sci 2006;51:881-981.

[19] Skripnyuk VM, Rabkin E, Estrin Y, Lapovok R. The effect of ball-milling and equal channel angular pressing on the hydrogen absorption/desorption properties of $\mathrm{Mg}-4.95 \mathrm{wt} \%$ Zn-0.71 wt\% Zr (ZK60) alloy. Acta Mater 2004;52:405-14.

[20] Kusadome Y, Ikeda K, Nakamori Y, Orimo S, Horita Z. Hydrogen storage capability of $\mathrm{MgNi}_{2}$ processed by high pressure torsion. Scripta Mater. 2007;57:751-3.

[21] Edalati K, Yamamoto A, Horita Z, Ishihara T. High pressure torsion of pure magnesium. Scripta Mater 2011;64:880-3.

[22] Huot J. Nanocrystalline metal hydrides obtained by severe plastic deformation. Metals 2012;2:22-40.

[23] Bellemare J, Huot J. Hydrogen storage properties of cold rolled magnesium hydride with oxides. J Alloys Compds 2012;512:33-8.

[24] Vincent SD, Huot J. Effect of air contamination on ball milling and cold rolling of magnesium hydride. J Alloys Compds 2012;509:L175-9.

[25] Leiva DR, de Almeida Costa HC, Huot J, Pinheiro TS, Jorge AM, Ishikawa TT, Botta WJ. Magnesium-nickel alloy for hydrogen storage produced by melt spinning followed by cold rolling. Materials Research 2012;15:813-7. 
[26] Skripnyuk VM, Rabkin E, Estrin Y, Lapovok R. Improving hydrogen storage properties of magnesium based alloys by equal channel angular pressing. Int J Hydrogen Energy 2009;34:6320-4.

[27] Skripnyuk V, Buchman E, Rabkin E, Estrin Y, Popov M, Jorgensen S. The effect of equal channel angular pressing on the hydrogen storage properties of a eutectic $\mathrm{Mg}-\mathrm{Ni}$ alloy. $\mathrm{J}$ Alloys Compds 2007;436:99-106.

[28] Jorge AM, Prokofiev E, de Lima GF, Rauch E, Veron M, Botta WJ, Kawasaki M, Langdon TG. An investigation of hydrogen storage in a magnesium-based alloy processed by equal-channel angular pressing. Int J Hydrogen Energy 2013;38:8306-12.

[29] Ribárik G, Gubicza J, Ungár T. Correlation between strength and microstructure of ballmilled Al-Mg alloys determined by X-ray diffraction. Mat Sci Eng A 2004;387-389:343-7.

[30] Révész Á, Fátay D. Microstructural evolution of ball-milled $\mathrm{MgH}_{2}$ during a complete dehydrogenation-hydrogenation cycle. J Power Sources 2010;195:6997-7002.

[31] Révész Á, Gajdics M, Spassov T. Microstructural evolution of ball-milled Mg-Ni powder during hydrogen sorption. Int J Hydrogen Energy 2013;38:8342-49.

[32] http://www.renyi.hu/cmwp

[33] Gennari FC, Esquivel MR. Structural characterization and hydrogen sorption properties of nanocrystalline $\mathrm{Mg}_{2} \mathrm{Ni}$. J Alloys Compd 2008;459:425-32.

[34] Bachmaier A, Hafok M, Schuster R, Pippan R. Limitations in the refinement by severe plastic deformation: The effect of processing. Rev Adv Mater Sci 2010;25:16-22. 


\section{Figures}

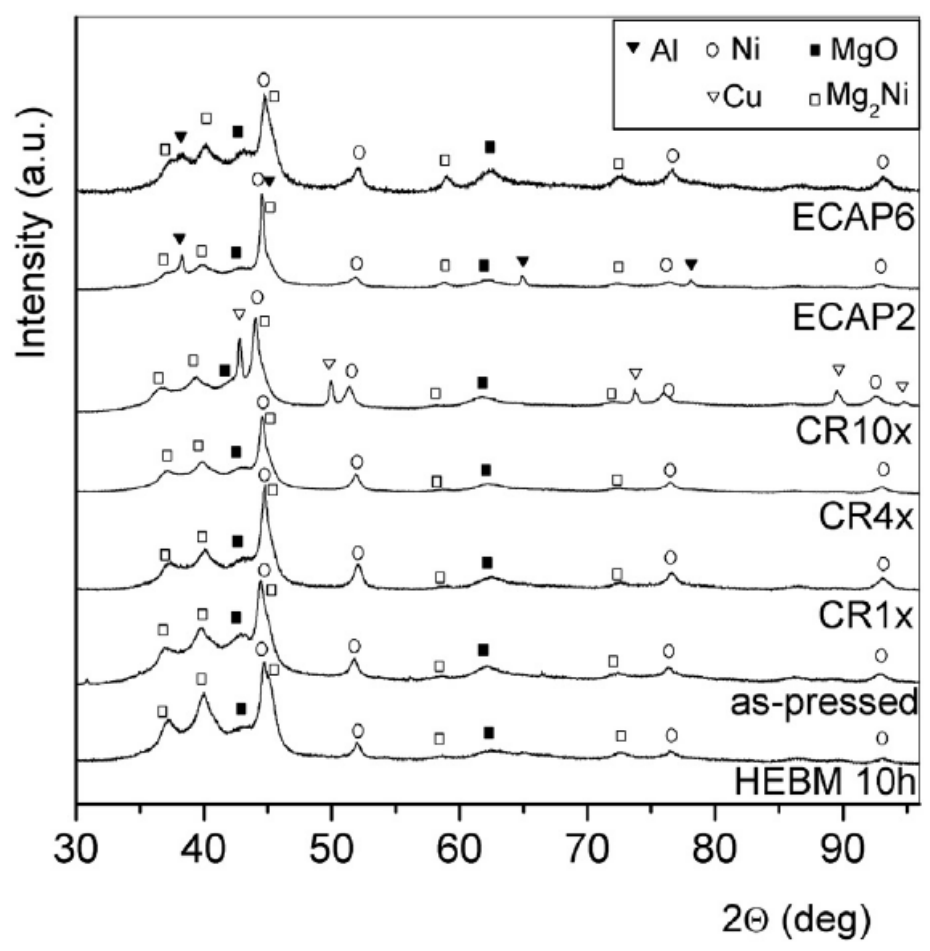

Fig 1. XRD patterns of the $\mathrm{Mg}_{2} \mathrm{Ni}$ powders processed by the combination of HEBM and ECAP or CR for different number of passes.

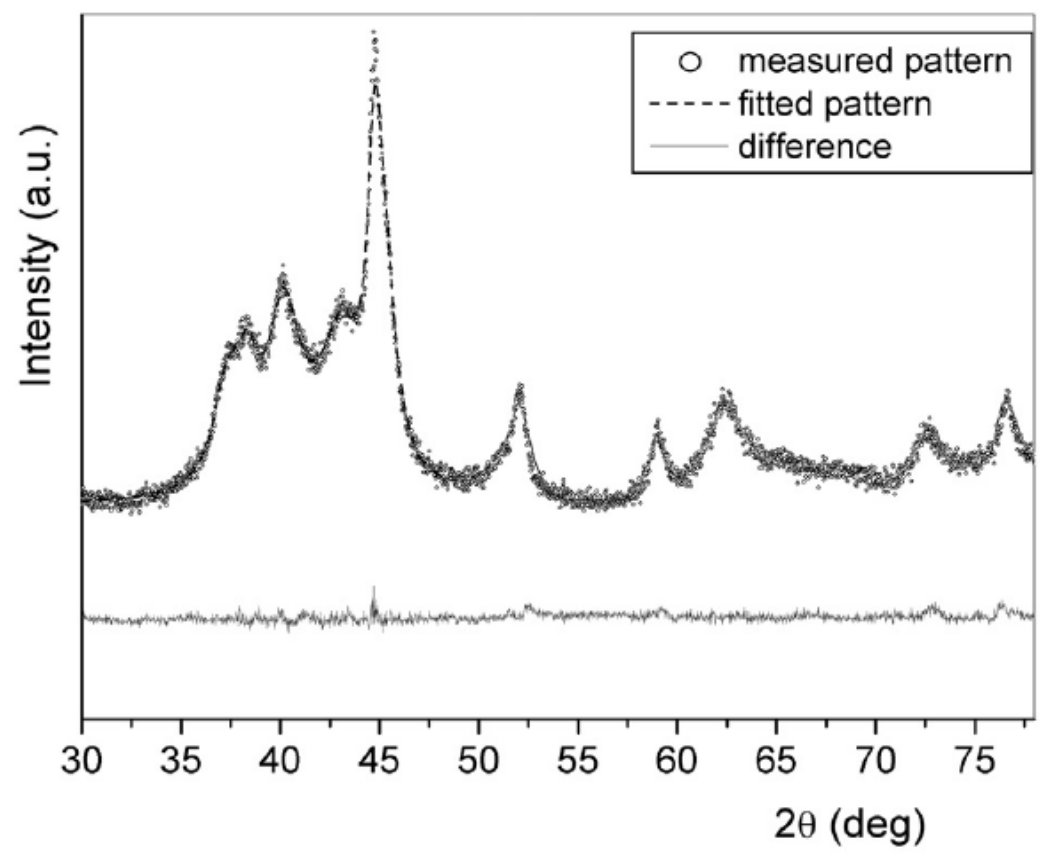

Fig 2.Fitted XRD pattern of the ECAP 6x sample by the CMWP method. The difference between the measurement and fit is also plotted. 

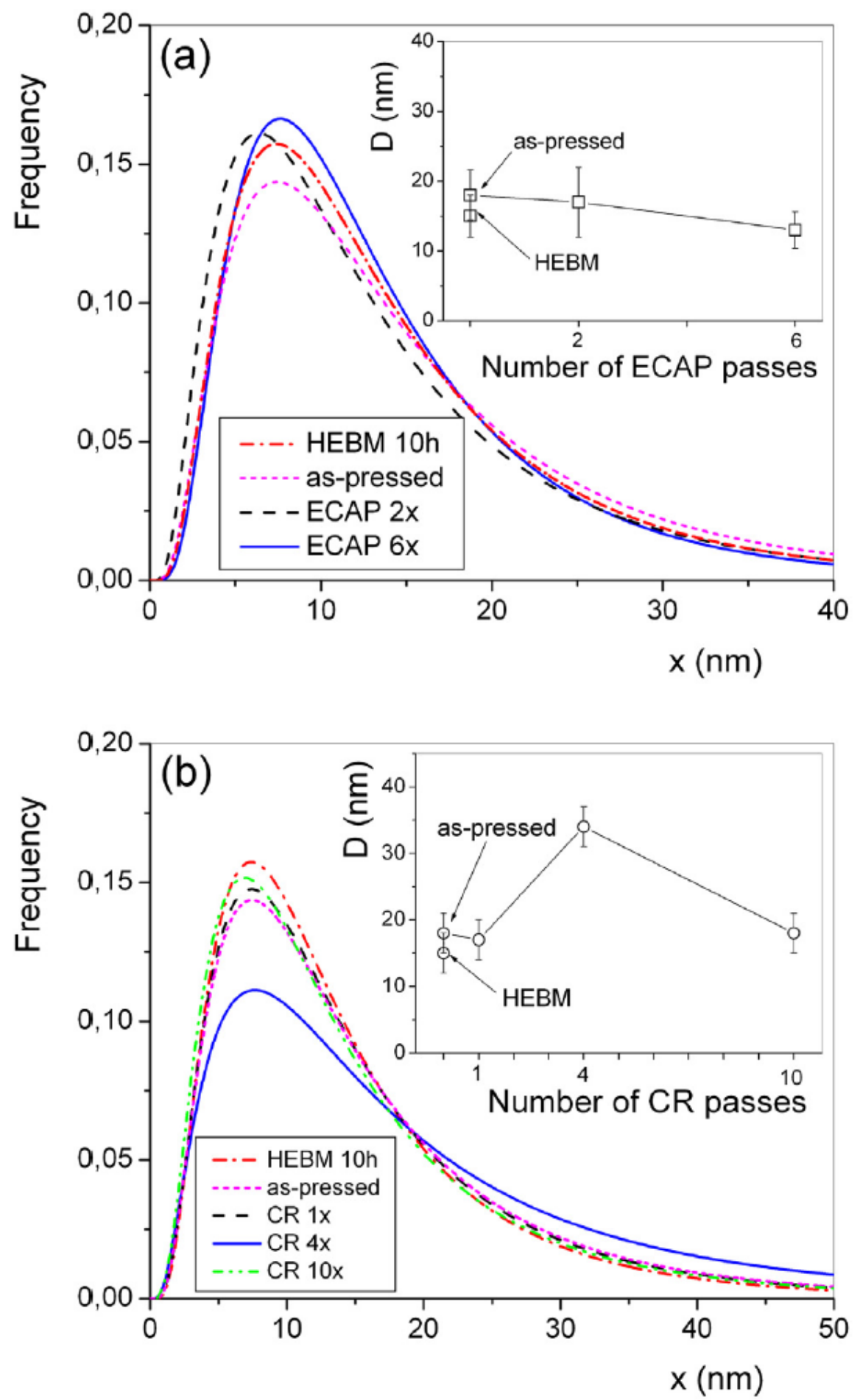

Fig 3 . Crystallite size histograms of the $\mathrm{Mg}_{2} \mathrm{Ni}$ phase obtained from the CMWP method of the (a) ECAP and (b) CR processed samples. Insets present the variation of the average coherent crystallite size (D) as a function of the ECAP and CR passes, respectively. 

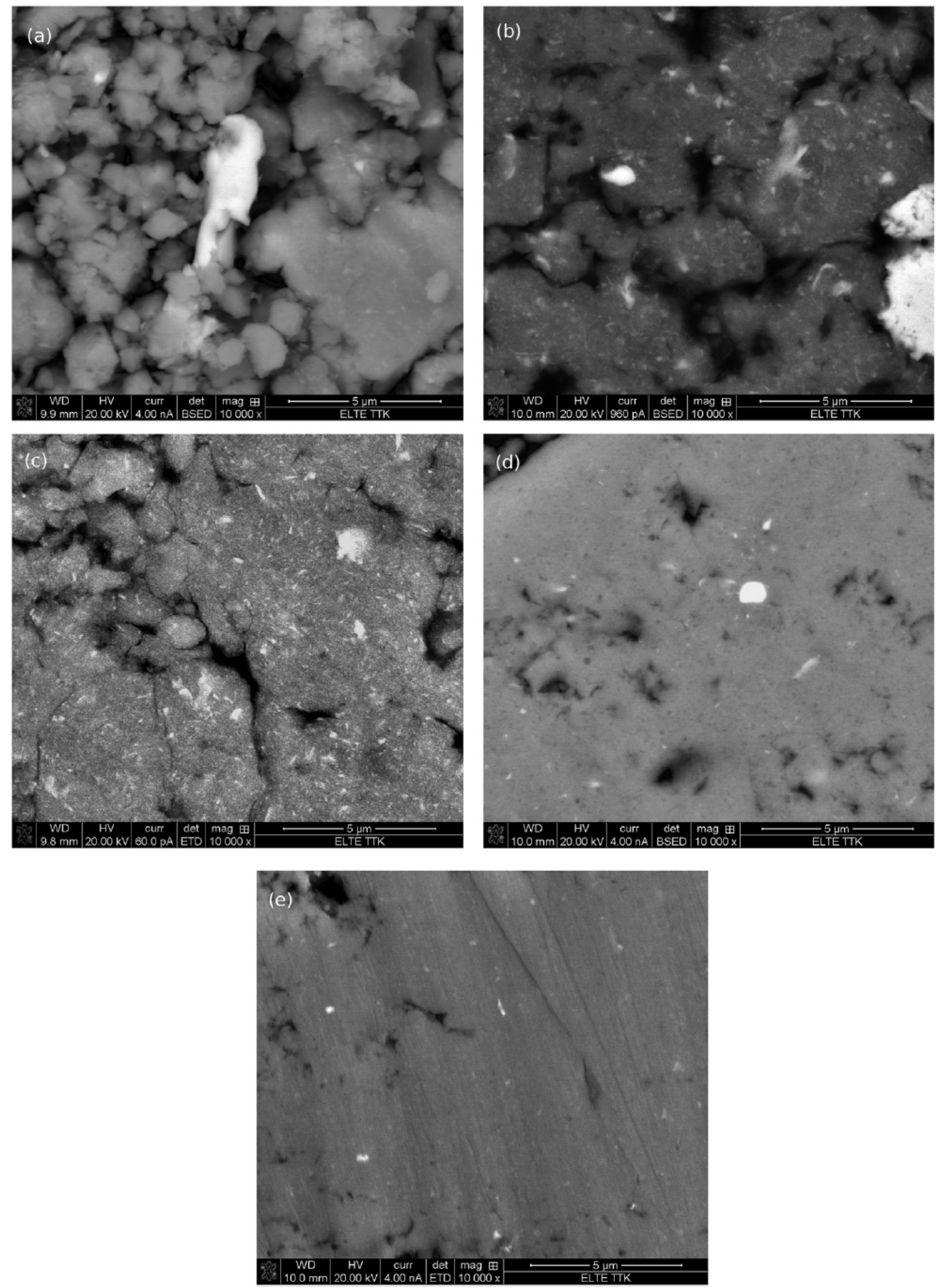

Fig 4. SEM images of the (a) as-milled $\mathrm{Mg}_{2} \mathrm{Ni}$ powder followed by (b) CR 1x, (c) CR 4, (d) ECAP 2x and (e) ECAP 6x passes. 


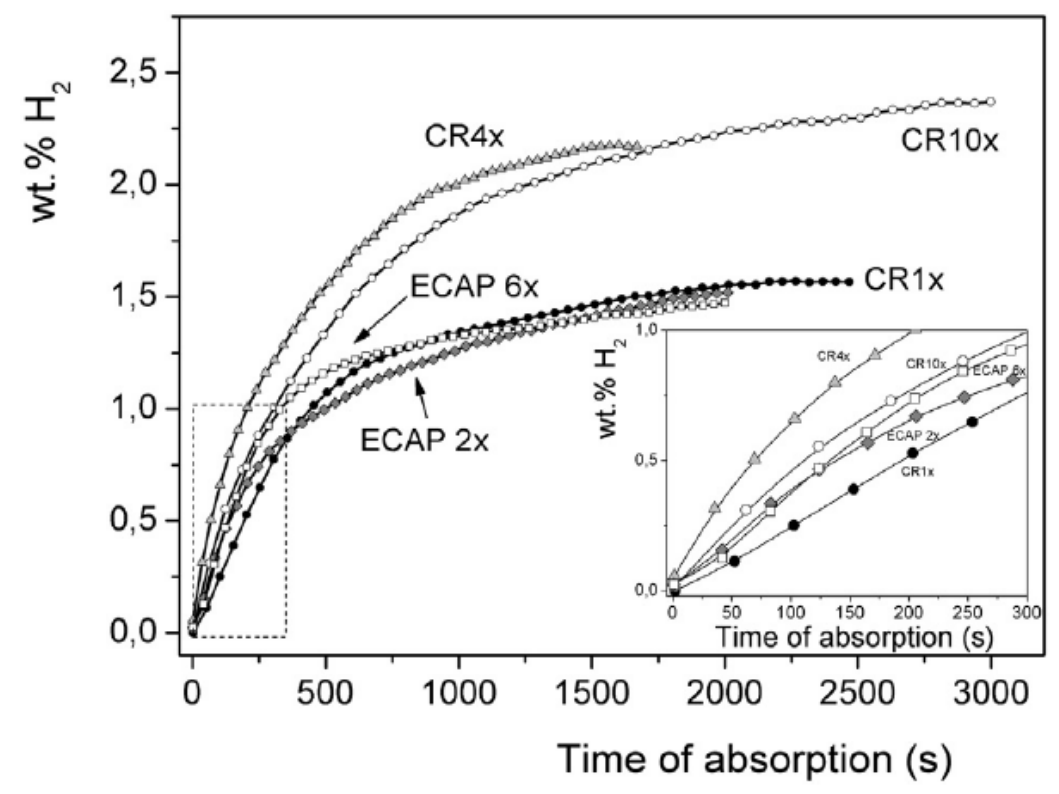

Fig 5. Hydrogen absorption curves of the ECAP and CR processed $\mathrm{Mg}_{2} \mathrm{Ni}$ samples. Inset shows the start of the transformations. 

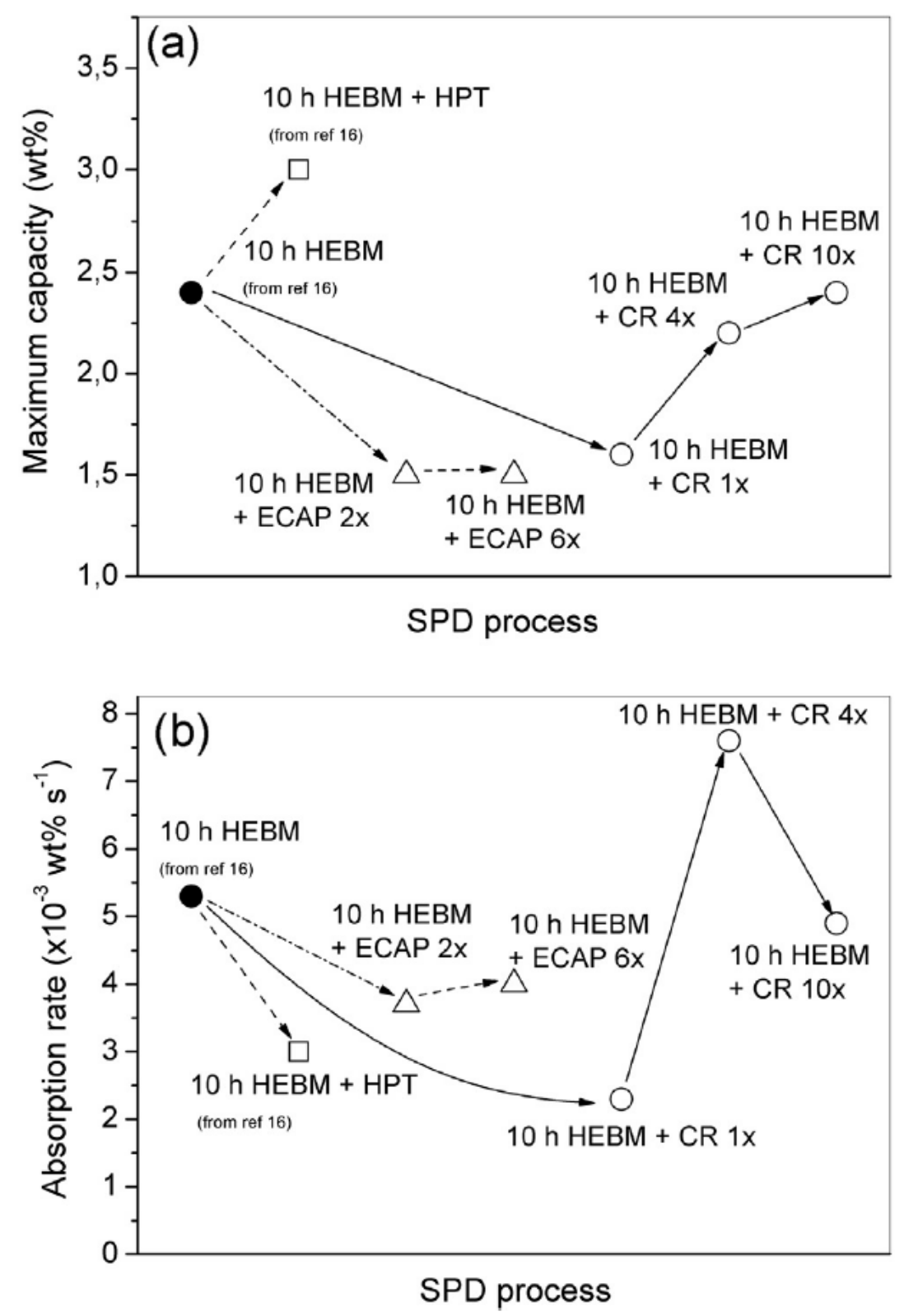

Fig 6. (a) Maximum hydrogen storage capacity and (b) initial absorption rate of $\mathrm{Mg}_{2} \mathrm{Ni}$ alloys processed by different SPD methods. 